Research Article

\title{
Efficacy of artemether-lumefantrine for the treatment of uncomplicated Plasmodium falciparum malaria in Northeast Ethiopia
}

\author{
Assefa Mulu $^{1 *}$, Berhanu Geresu ${ }^{1}$, Yeshiwork Beyene ${ }^{2}$, Muluneh Ademe ${ }^{3}$
}

\begin{abstract}
${ }^{1}$ Department of Pharmacy, Wollo University, Dessie, Ethiopia, ${ }^{2}$ Department of Nursing, Wollo University, Dessie, Ethiopia, ${ }^{3}$ Department of Microbiology, Immunology and Parasitology, Addis Ababa University, Addis Ababa, Ethiopia

Received: 10 March 2015 Accepted: 24 April 2015

*Correspondence to: Mr. Assefa Mulu, Email: mulubaye@gmail.com

Copyright: (C) the author(s), publisher and licensee Medip Academy. This is an openaccess article distributed under the terms of the Creative Commons Attribution NonCommercial License, which permits unrestricted noncommercial use, distribution, and reproduction in any medium, provided the original work is properly cited.
\end{abstract}

\begin{abstract}
Background: The impact of resistance to antimalarials is insidious and unless efficacy studies are conducted, resistance may go unrecognized. The aim of this study was to assess the efficacy of artemether/lumefantrine, for the treatment of uncomplicated Plasmodium falciparum infections in Kemisie Health Center, Northeast Ethiopia. Methods: Artemether/lumefantrine efficacy study was conducted in Kemisie Health Center, Northeast Ethiopia from September, 2012 to May, 2013. The study participants were febrile people above the age of 6 months with confirmed uncomplicated $P$. falciparum infection. Patients were treated with artemether/lumefantrine. Clinical and parasitological parameters were monitored over a 28 days follow-up period to evaluate drug efficacy. The Kaplan-Meier method was used for statistical analysis of data on drug efficacy.

Results: Among the 66 enrolled participants $72.7 \%$ were 15 and above years of age while $15.3 \%$ were less than 5 years old. Of these study participants, $53 \%$ were male. There was $89.4 \%$ fever clearance and $84.8 \%$ parasite clearance on day 1 , whereas, there was $100 \%$ fever clearance and $96.8 \%$ parasite clearance on day 3 from the 62 participants who carried on the study. Among 66 participants who had started 28 days of follow-up one patient had an early parasitological failure (parasitemia on day 3) and another patient had a late parasitological failure (parasitemia on day 21). Conclusions: In general, this study discovered good clinical and parasitological response of $P$. falciparum to artemether/lumefantrine. Further polymeric chain reaction and plasma drug concentration based effectiveness study of artemether/ lumefantrine should be conducted throughout the country.
\end{abstract}

Keywords: Artemether/lumefantrine, Plasmodium falciparum malaria, Efficacy, Ethiopia

\section{INTRODUCTION}

Malaria is a leading cause of morbidity and mortality in developing areas of the world and remains a major health problem in endemic regions. It is estimated that 300500 million clinical cases occur every year and that over two million people die every year from the disease. ${ }^{1}$

In Ethiopia, the increased resistance of Plasmodium falciparum to chloroquine and sulfadoxine-pyrimethamine necessitated a change as a first-line antimalarial drug for the treatment of $P$. falciparum. Consequently, artemether/ lumefantrine $\left(\right.$ Coartem $\left.^{\circledR}\right)$ was adopted in 2004. Currently, the drug is being used as the first-line drug for the treatment of uncomplicated malaria. ${ }^{2}$
As the trend of malaria changes over time, the efficacy of the recommended treatments shall be monitored. Emphasis should particularly be given to monitoring the emergence of resistance to the anti-malarial drugs in use..$^{2,3}$

Resistance to antimalarial medicines has been documented in all classes of antimalarials, including the artemisinin derivatives, and it is a major threat to malaria control. ${ }^{4}$

Reports of artemisinin resistance from the Thai-Cambodian border, the epicenter of drug resistance, raise global concern for the long-term efficacy of artemisinin-based combination therapy (ACT). ${ }^{5,6}$

A 28 days therapeutic efficacy study of artemether/ lumefantrine against $P$. falciparum conducted between 2007 
and 2008 in Kersa, Southwest Ethiopia proved that cure rate was very high, $96.3 \%$, adequate clinical and parasitological response (ACPR) to be $100 \%$ for children under 5 and $97.4 \%$ and $87.3 \%$ for children aged $5-14$, and adults, respectively. There was no early treatment failure in all age groups. ${ }^{7}$

Eight years have passed since artemether/lumefantrine was adopted as first-line therapy in Ethiopia, which coincided with the Federal Ministry of Health's (FMoH's) ambitious plan to provide universal access to prompt malaria diagnosis and treatment - as recently recommended by World Health Organization (WHO) ${ }^{8}$ WHO generally recommends that the efficacy of first- and second-line medicines be tested at least once every 24 months at all sites. ${ }^{9}$ Since data on the efficacy of ACT in Ethiopia is limited, we found that the finding of this study is essential to generate a baseline data so that the FMoH of Ethiopia will take informed decision regarding antimalarial treatment guidelines.

\section{METHODS}

\section{Study design}

This surveillance study is a one-arm prospective evaluation of clinical and parasitological responses to directly observed treatment for uncomplicated malaria. The WHO protocol "methods for surveillance of antimalarial drug efficacy" was used. ${ }^{\text {" }}$

\section{Study settings}

The study was conducted in Kemisie Health Center, which serves the town and the neighboring Kebeles. The health center is found in Kemisie Town of Oromiya zone, Amhara Region, Northeast Ethiopia. Both P. falciparum and Plasmodium vivax are endemic to this area. The health center is located at an altitude of $1424 \mathrm{~m}$ above sea level which is favorable for malaria transmission. The study was conducted during September, 2012 to May, 2013.

\section{Study participants}

As this is a single-arm efficacy study, sample size determination was based on an expected proportion of treatment failures in the study population and the desired levels of confidence (95\%) and precision (5\%). Taking a failure rate of $3.7 \%$ that leads to the maximum sample size by a previous study at Asendabo, Southwest Ethiopia (7), a confidence level of $95 \%$ and a precision level of $5 \%$; the sample size was calculated to be 66 patients including additional $20 \%$ patients to account for those who are likely to be either lost during follow-up or withdraw as recommended by WHO. ${ }^{9}$

The study participants consisted of patients with uncomplicated $P$. falciparum malaria attending the study health clinic that were above 6 months of age. Other inclusion criteria were monoinfection with $P$. falciparum detected by microscopy; parasitemia of $1000-1,00,000 / \mu 1$ asexual forms; axillary temperature $\geq 37.5^{\circ} \mathrm{C}$ or history of fever during the past $24 \mathrm{hrs}$; and able to swallow oral medications. Whereas, patients with severe malnutrition, febrile conditions due to diseases other than malaria and other known underlying chronic or severe diseases, regular medication, which may interfere with antimalarial pharmacokinetics, history of hypersensitivity reactions or contraindications to any of the medicines being tested; a positive pregnancy test or breastfeeding, unable to comply with the study protocol were excluded.

\section{Clinical and laboratory procedures}

Artemether-lumefantrine was administered as of a tablet containing $20 \mathrm{mg}$ of artemether and $120 \mathrm{mg}$ of lumefantrine. The dosing was based on the number of tablets per dose according to predefined weight bands (5-14 kg: 1 tablet; 15-24 kg: 2 tablet s; 25-34 kg: 3 tablets; and >34 kg: 4 tablets), given twice a day for 3 days. Tablets of artemether/ lumefantrine $(20 / 120 \mathrm{mg})$ were obtained from Novartis Pharma, Basel, Switzerland through WHO to Ministry of Health, Ethiopia.

Female patients of child-bearing age, defined as those who menstruate or were aged over 12 years, were asked to take a urine pregnancy test before enrolment in the study, because artemether/lumefantrine is contraindicated during the first trimester. They were asked to take a urine pregnancy test on day 28 or on early withdrawal from the study.

A standard physical examination were performed at baseline (day 0 before dosing) and on days 1, 2, 3, 7, 14, 21, and 28 . Axillary temperature was measured at baseline (day 0 before dosing) and on days 1, 2, 3, 7, 14, 21, and 28 .

Thick and thin blood films for parasite counts were obtained and examined at screening on day 0 to confirm adherence to the inclusion and exclusion criteria. Thick blood films were also examined on days $2,3,7,14,21$, and 28 .

The blood smear was used to calculate the parasite density, by counting the number of asexual parasites in a set number of white blood cells (typically 200) with a hand tally counter. When more than 500 parasites had been counted before 200 white blood cells had been reached, the count was stopped after the reading of the last field has been completed. Parasite density was calculated as:

$$
\begin{aligned}
& \text { Parasite density }(\text { per } \mu 1)=(\text { number of parasites counted }) \\
& \times \frac{8000}{\text { Numberof leukocytes counted }}
\end{aligned}
$$

Two qualified medical laboratory technicians read all the slides independently, and parasite densities were calculated by averaging the two counts. 


\section{Treatment outcomes}

Treatment outcomes were classified on the basis of an assessment of the parasitological and clinical outcome of antimalarial treatment according to the latest WHO guidelines. ${ }^{9}$ Thus, all patients were classified as having an early treatment failure, late clinical failure, late parasitological failure or an ACPR.

\section{Data processing and analysis}

Data analysis was conducted using SPSS version 20.0. The Kaplan-Meier method was used for statistical analysis of data on drug efficacy. All the baseline and follow-up data collected on a patient until the day of censoring - the last day the patient was observed and was not classified as a failure - were included in the analysis, even for patients who did not complete the study.

\section{RESULTS}

\section{Screening, admission and follow-up of patients}

Patients who were febrile or had a history of fever with in past $24 \mathrm{hrs}$ of diagnosis were sent from outpatient department to laboratory and diagnosed for malaria. During the study period, 2542 febrile cases and other clinically suspected to be infected with malaria were tested for the presence of the parasite. Of these febrile cases, $253(10.2 \%)$ had malaria positive slides and $109(43.1 \%)$ of them had P. falciparum mono-infections. Of those $P$. falciparum mono-infected patients, 6 could not be enrolled in the study for having a parasite load below 1000 parasites per microliter of blood. Of the rest 103 patients, 37 were excluded for the following reasons: 29 were out of the reachable area, 5 were pregnant, and another 3 took antimalaria drugs before initiation of the study.

Totally, 66 patients fulfilled all the criteria and enrolled in the study but 6 were excluded due to their failure to attend the scheduled visits ( 3 at day 2, 1 at day 3, and 2 at day 21). Two patients were excluded at the $3^{\text {rd }}$ and $21^{\text {st }}$ day as they were found to have treatment failure.

\section{Socio-demographic characteristics}

Among the 66 enrolled participants, $72.7 \%$ were 15 and above years of age while $15.3 \%$ were less than 5 years of old. Of these study participants, $53 \%$ were male. The sociodemographic characteristics of the study participants are displayed in Table 1.

\section{Clinical characteristics}

Among the study participants, body temperature had a range from 37 to $39^{\circ} \mathrm{C}$ and parasite load varies from 1080 to 29640 count/ $\mu 1$ on the day of enrollment. Explanatory results are presented in Table 2.
In age and sex distribution of clinical characteristics, relatively higher geometric mean parasite load and mean axillary temperature was documented in under five age group while relatively lower in the age of 15 and above as indicated by Table 3 .

Table 1: Socio-demographic characteristics of the study participants on the day of enrollment, Kemissie Health Center, Northeast Ethiopia, 2013.

\begin{tabular}{|lc|}
\hline Characteristics & $\begin{array}{c}\text { Value at day of } \\
\text { admission (day } \mathbf{0})(\%)\end{array}$ \\
\hline Age groups (years) & $10(15.26)$ \\
\hline$<5$ & $8(12.1)$ \\
\hline $5-14$ & $48(72.7)$ \\
\hline 15 above \\
\hline Sex & $31(47.0)$ \\
\hline Female & $35(53.0)$ \\
\hline Male & 1.45 \\
\hline Mean height $(\mathrm{cm})$ & 46.02 \\
\hline Mean weight $(\mathrm{kg})$ & \\
\hline
\end{tabular}

Table 2: Clinical characteristics of the study participants on the day of enrollment, Kemissie Health Center, Northeast Ethiopia; 2013.

\begin{tabular}{|lc|}
\hline Characteristics & $\begin{array}{c}\text { Value at day of } \\
\text { admission (day 0) }\end{array}$ \\
\hline $\begin{array}{l}\text { Mean body temperature } \\
\text { in degree celsius } \pm \text { SD }\end{array}$ & $38.17( \pm 0.3748)$ \\
\hline History of fever $(\mathrm{N} / \%)$ & $59(89.4)$ \\
\hline $\begin{array}{l}\text { Geometric mean initial } \\
\text { parasite } / \mu \mathrm{l} \pm \mathrm{SD}\end{array}$ & $10454( \pm 5670)$ \\
\hline
\end{tabular}

SD: Standard deviation

Table 3: Baseline clinical characteristics of participants stratified by age and sex, in Kemisie

Health Center, Northeast Ethiopia, 2013.

\begin{tabular}{|c|c|c|c|c|}
\hline \multirow[t]{2}{*}{$\begin{array}{l}\text { Age in } \\
\text { year }\end{array}$} & $\operatorname{Sex}$ & Mean body & $\begin{array}{l}\text { History of } \\
\text { fever }\end{array}$ & $\begin{array}{c}\text { Geometric } \\
\text { mean }\end{array}$ \\
\hline & & $\begin{array}{c}\text { Temperature } \\
\left({ }^{\circ} \mathrm{C}\right)\end{array}$ & $\begin{array}{l}\text { Within past } \\
24 \mathrm{hrs} / \%\end{array}$ & Parasite/ $\mu \mathrm{L}$ \\
\hline \multirow[t]{2}{*}{$<5$} & Male & 38.48 & 100 & 17505 \\
\hline & Female & 38.30 & 100 & 13823 \\
\hline \multirow[t]{2}{*}{$5-14$} & Male & 38.14 & 100 & 11697 \\
\hline & Female & 38.35 & 100 & 9943 \\
\hline \multirow[t]{2}{*}{$>14$} & Male & 38.12 & 92.6 & 9446 \\
\hline & Female & 38.09 & 76.2 & 9307 \\
\hline \multirow[t]{2}{*}{$\begin{array}{l}\text { All age } \\
\text { group }\end{array}$} & Male & 38.17 & 94.3 & 10625 \\
\hline & Female & 37.17 & 83.9 & 10263 \\
\hline Total & & 38.17 & 89.4 & 10454 \\
\hline
\end{tabular}




\section{Fever and parasite clearance rate}

As indicated in Table 4, there was $89.4 \%$ fever clearance and $84.8 \%$ parasite clearance on day 1 . Whereas, on day 2 there was $98.5 \%$ fever clearance $93.9 \%$ parasite clearance among 63 participants who continued in the study. There was $100 \%$ fever clearance $96.8 \%$ and parasite clearance on day 3 from the 62 participants who carried on the study.

\section{Treatment outcome}

Among 66 participants who had started 28 days of follow-up one patient had an early parasitological failure (parasitemia on day 3) and another patient had a late parasitological failure (parasitemia on day 21) (Table 5). A 25-year-old female participant had recurrent parasitemia on day 3 with parasite load of 5100/ $\mu 1$ and a 6-year-old male patient had recurrence parasitemia on day 21 with asexual parasite load of $2300 / \mu 1$. Both of them had the clinical symptom of malaria at the day of recurrence.

According to Kaplan-Meier analysis the total cumulative incidence of treatment failure was $3.28 \%$ (95\% confidence interval: $1.32,5.24)$. As the exclusion of the four participants from the study (on day 2 and day 3 ) was before treatment failure (on day 3 ) censoring of these participants did not alter the cumulative incidence of treatment failure. However, the exclusion of two participants from the study (on day 21) was after treatment failure (on day 3 ) where censoring of these participants altered the cumulative incidence of treatment failure. Exclusion from the study was due to loss to followup in all six participants.

\section{DISCUSSION}

Artemether/lumefantrine clears fever and parasitemia caused by $P$. falciparum within, at most, 72 hrs of the first dose. Previously, it was $100 \%$ effective in the treatment of P. falciparum. ${ }^{4}$ This study revealed $96.72 \%$ effectiveness of the drug at the end of 28 days follow-up.
In this study, at the day of enrollment $89.4 \%$ of patients were febrile and $98.5 \%$ cleared within $48 \mathrm{hrs}$ and $100 \%$ within $72 \mathrm{hrs}$ of the follow-up. Parasite clearance rate was $93.9 \%$ on day 2 and $96.8 \%$ on the day 3. This is similar with studies which were conducted in Kersa district ${ }^{7}$ and central Ethiopia ${ }^{10}$ in which almost all patients were cleared of parasites on the day 3 .

In this study, there were one early parasitological failure and one late parasitological failure in a 25 -year-old female participant and a 6-year-old male participant. The female patient on the day of enrollment had an axillary temperature of $38.2^{\circ} \mathrm{C}$ and $2500 / \mu$ l of parasite load. Fever cleared within $48 \mathrm{hrs}$ where parasite load decreased to 7200 count/ $\mu$ l on day 2 and persist to day 3 with a parasite load of $5100 \mathrm{count} / \mu 1$.

In the 6-year-old male patient, on the day of enrollment, he had an axillary temperature of $38.9^{\circ} \mathrm{C}$ and fever were cleared within 24 hrs. Parasite load on enrollment was 24460 count $/ \mu 1$ and he was cleared of parasitemia on day 3. Despite fast clearance of fever and parasitemia, there was reappearance of parasitemia on day 21 without a clinical symptom of malaria.

According to Kaplan-Meier analysis, in the case of loss to follow-up and withdrawn from study, participants should be excluded from study but for analysis, censored last day of follow-up according to timetable for lost to follow-up and censored last day of follow-up according to timetable before withdrawal or protocol violation for withdrawals.

In this study, six participants were lost to follow-up despite of every effort (three on day 2 and one on day 3 and two on day 21 of follow-up). The two treatment failures occurred on day 3 and on day 21. Therefore, three participants on day 2 , two participants on day 3 and three participants on day 21 were excluded from the study. For the treatment failure that occurred on day 3 censoring for withdrawal do not affect the cumulative incidence of treatment failure. However, for the treatment failure that occurred on day 21 censoring for withdrawal affect the cumulative incidence of treatment failure. Therefore, our cumulative incidence of treatment failure was $3.27 \%$.

Table 4: Fever and parasite clearance rate (\%) of the study participants stratified by age and sex, Kemisie Health Center Northeast Ethiopia, 2013.

\begin{tabular}{|llcccccc|}
\hline $\begin{array}{l}\text { Age } \\
\text { group }\end{array}$ & Sex & \multicolumn{2}{c}{ Day 1 } & \multicolumn{2}{c}{ Day 2 } & \multicolumn{2}{c|}{ Day 3 } \\
\hline$<5$ & & Fever & Parasite & Fever & Parasite & Fever & Parasite \\
\hline & Male & 100 & 50.0 & 100 & 75 & 100 & 100 \\
\hline $5-14$ & Female & 66.7 & 66.7 & 83.3 & 100 & 100 & 100 \\
\hline & Male & 100 & 75.0 & 100 & 75 & 100 & 66.7 \\
\hline$>14$ & Female & 100 & 100 & 100 & 100 & 100 & 100 \\
\hline & Male & 92.6 & 88.9 & 100 & 96.3 & 100 & 100 \\
\hline All groups & Female & 90.5 & 90.5 & 100 & 95.2 & 100 & 95 \\
\hline & Male & 94.3 & 82.9 & 100 & 94.1 & 100 & 97.1 \\
\hline Total & Female & 87.1 & 87.1 & 96.8 & 96.8 & 100 & 96.6 \\
\hline
\end{tabular}


Table 5: Treatment outcome of study participants versus days of follow-up, in Kemisie Health Center, Northeast Ethiopia, 2013.

\begin{tabular}{|lcccccc|}
$\begin{array}{l}\text { Follow-up } \\
\text { days }\end{array}$ & TF & W & PR & $\begin{array}{c}\text { II } \\
(\%)\end{array}$ & $\begin{array}{c}\text { CITF } \\
(\%)\end{array}$ & 95\% CI \\
\hline Day 0 & - & - & 66 & - & - & - \\
\hline Day 1 & 0 & 0 & 66 & 0 & 0 & - \\
\hline Day 2 & 0 & 3 & 63 & 0 & 0 & $-3.40,3.40$ \\
\hline Day 3 & 1 & 1 & 62 & 1.61 & 1.61 & $-0.35,3.57$ \\
\hline Day 7 & 0 & 0 & 61 & 0 & 1.61 & $-0.35,3.57$ \\
\hline Day 14 & 0 & 0 & 61 & 0 & 1.61 & $-0.35,3.57$ \\
\hline Day 21 & 1 & 2 & 59 & 1.695 & 3.28 & $1.32,5.24$ \\
\hline Day 28 & 0 & 0 & 58 & 0 & 3.28 & $1.32,5.24$ \\
\hline
\end{tabular}

PR: Population at risk, W: Excluded from the study, TF: Treatment failure, II: Interval incidence, CI: Confidence interval, LL: Lower limit, UL: Upper limit, CI describes range of probability under which cumulative incidence of treatment failure may fall

Recurrence parasitemia may be due to various reasons; recrudescence, reinfection or resistance. The day 3 recurrence is most probably resistance strain and less probably reinfection, because plasma therapeutic concentration sustains more than 21 days after three-day standard dosage of artemether/lumefantrine. Even if it is reinfection, it is most probably resistance strain. However, our study lacks test of the therapeutic level of artemether/lumefantrine in the blood to conclude accordingly. Recurrence on day 21 may be due to any of above mentioned reasons which can only be determined genotyping in addition to blood drug concentration. Unfortunately, our study puts up with limitation of the absence of genotyping of $P$. falciparum species.

The findings of this study showed that artemether/ lumefantrine maintained as an efficacious regimen in Kemisie Health Center. The high cure rate illustrated in this study is consistent with recent studies in Ethiopia ${ }^{7,10}$ with cure rates of $93.3 \%$ and $96.3 \%$, respectively, and studies from somewhere else in Africa. ${ }^{11}$ The cure rate for this study at day 28 was $96.72 \%$. It is also in parallel with the global study of antimalarial drug efficacy and drug resistance between 2000 and $2010 .{ }^{12}$

\section{CONCLUSIONS}

In general, this study discovered good clinical and parasitological response of $P$. falciparum to artemether/ lumefantrine. Our study also showed fast clearance of fever and parasitemia within maximum of $72 \mathrm{hrs}$ of the start of medication. With reported response rate, the artemether/ lumefantrine can be continued as first line medication for uncomplicated $P$. falciparum malaria in the study area. Further polymeric chain reaction and plasma drug concentration based effectiveness study of artemether/ lumefantrine should be conducted throughout the country.

\section{ACKNOWLEDGMENTS}

We acknowledge Wollo University for funding this study. We are very grateful for the study participants for their patience to complete the study. We are indebted to thank the staffs of the Kemisie Health Center for their cooperation during the study.

\section{Funding: The research was funded by Wollo University Conflict of interest: None declared \\ Ethical approval: The study has got ethical clearance from Wollo University Ethical Review Committee}

\section{REFERENCES}

1. Wernsdorfer WH. Epidemiology of drug resistance in malaria. Acta Trop. 1994;56(2-3):143-56.

2. FMOH (Federal Republic Ethiopia, Ministry of Health). Malaria diagnosis and treatment a guideline for health workers in Ethiopia. Addis Ababa: FMOH; 2004.

3. Haque R, Thriemer K, Wang Z, Sato K, Wagatsuma Y, Salam MA, et al. Short report: therapeutic efficacy of artemether-lumefantrine for the treatment of uncomplicated plasmodium falciparum malaria in Bangladesh. Am J Trop Med Hyg. 2007;76(1):39-41.

4. WHO. Guidelines for the Treatment of Malaria. Geneva: World Health Organization; 2006.

5. Dondorp AM, Nosten F, Yi P, Das D, Phyo AP, Tarning J, et al. Artemisinin resistance in Plasmodium falciparum malaria. N Engl J Med. 2009;361(5):455-67.

6. Noedl H, Se Y, Schaecher K, Smith BL, Socheat D, Fukuda MM, et al. Evidence of artemisinin-resistant malaria in western Cambodia. N Engl J Med. 2008;359(24):2619-20.

7. Assefa A, Kassa M, Tadese G, Mohamed H, Animut A, Mengesha T. Therapeutic efficacy of Artemether/ Lumefantrine (Coartem(R)) against Plasmodium falciparum in Kersa, South West Ethiopia. Parasit Vectors. 2010;3(1):1.

8. WHO. Guidelines for the Treatment of Malaria. 2nd Edition Geneva: World Health Organization; 2010.

9. WHO. Methods for Surveillance of Antimalarial Drug Efficacy. Geneva: World Health Organization; 2009.

10. Hwang J, Alemayehu BH, Hoos D, Melaku Z, Tekleyohannes SG, Teshi $\mathrm{T}$, et al. In vivo efficacy of artemether-lumefantrine against uncomplicated Plasmodium falciparum malaria in Central Ethiopia. Malar J. 2011;10:209.

11. Mulenga M, VangGeertruyden JP, Mwananyanda L, Chalwe V, Moerman F, Chilengi R, et al. Safety and efficacy of lumefantrine-artemether (Coartem) for the treatment of uncomplicated Plasmodium falciparum malaria in Zambian adults. Malar J. 2006;5:73.

12. WHO. Global Report on Antimalarial Drug Efficacy and Drug Resistance: 2000-2010. Geneva: World Health Organization; 2010.

doi: $10.18203 / 2319-2003 . i j b c p 20150026$

Cite this article as: Mulu A, Geresu B, Beyene Y, Ademe M. Efficacy of artemether-lumefantrine for the treatment of uncomplicated Plasmodium falciparum malaria in Northeast Ethiopia. Int J Basic Clin Pharmacol 2015;4:492-6. 